\section{Evaluation of preprandial and two-hour postprandial serum bile acid concentrations in referral population of dogs}

\section{Veronica Gonzalo Nadal, Rodolfo Cappello, Alenka Hrovat}

North Downs Specialist Referrals, Bletchingley, United Kingdom

\section{OBJECTIVES}

To examine preprandial (PreSBA) and postprandial serum bile acid (PostSBA) concentrations in dogs referred for a work up of neurological, hepatobiliary disease and portosystemic shunts (PSS).

\section{METHODS}

All dogs with measured PreSBA and PostSBA concentrations were included retrospectively from 2014-2017. Dogs with increased bilirubin and incomplete BA test results were excluded. Dogs were divided into four groups based on the final diagnose, i.e. neurological, hepatobiliary disease, PSS and other.

\section{RESULTS}

182 dogs met the inclusion criteria. Primary neurological disease was diagnosed in 48/182 dogs. Idiopathic epilepsy was established in 29 and intracranial or spinal disease in 19 dogs with median PostSBA 3.4 (range, $0.8-$ $31.8 \mu \mathrm{mol} / \mathrm{L}$ ) and 12.1 (range, $0.2-60.5 \mu \mathrm{mol} / \mathrm{L}$ ), respectively. Hepatobiliary disease was diagnosed in $32 / 182$ dogs with PostSBA 19.2 (range, 0.1-147.5 $\mu \mathrm{mol} / \mathrm{L}$ ). PSS was diagnosed in 21/ 182 dogs with PostBA 140.2 (range, 43.5-283.3 $\mu \mathrm{mol} / \mathrm{L}$ ). 81 dogs presented with conditions other than hepatobiliary and neurological disease with median PostSBA 4.3 (range, 0.1-61.6 $\mu \mathrm{mol} / \mathrm{L}$ ). PreSBA and PostSBA concentrations in dogs with PSS were significantly higher $(p<0.01)$ than in dogs with primary neurological and hepatobiliary diseases. PreSBA and PostSBA concentrations in 19/21 dogs with PSS exceeded $75 \mathrm{\mu mol} / \mathrm{L}$. All dogs with primary neurological disease and idiopathic epilepsy had PreSBA and PostSBA concentrations below $75 \mathrm{\mu mol} / \mathrm{L}$.

\section{STATEMENT (CONCLUSIONS)}

Bile acid concentrations in dogs with neurological and hepatobiliary disorders are significantly lower than in dogs with PSS. Based on results of this study PSS is unlikely in dogs with PreSBA and PostSBA concentrations below $75 \mu \mathrm{mol} / \mathrm{L}$.

\section{Assessment of visceral pain and quality of life in dogs with chronic enteropathy - A controlled questionnaire based pilot study}

\section{Jorge Pérez-Accino, Amy Miele, Silke Salavati}

Royal (Dick) School of Veterinary Studies, Roslin, United Kingdom

\section{OBJECTIVES}

Assessing visceral pain in dogs diagnosed with chronic enteropathy (CE) and its relation to quality of life (QOL).

\section{METHODS}

Institutional veterinary and human ethic committees approved the study. Adult dogs presenting with chronic gastrointestinal (GI) signs to a referral hospital $(n=28)$ were prospectively enrolled and received a standard diagnostic work-up; 18/28 were diagnosed with CE and $10 / 28$ with suspected CE, lacking histopathology. A modified Canine Brief Pain Inventory ${ }^{\odot}$ (mCBPI) questionnaire was completed by the owners and a visceral pain score (modified Colorado State University Canine Chronic Pain Scale, mCSUCCPS) completed by a veterinarian. A group of control dogs $(n=22)$ was scored as well. Mann-Whitney $U$ test was used to assess differences in scores between cases and controls. Spearman's rank correlation was determined for specific parameters. Significance was set at $p<0.05$.

\section{RESULTS}

The $\mathrm{mCBPI}$ and $\mathrm{mCSUCCPS}$ scores were significantly higher in dogs with CE compared to controls ( $p=0.0001$, $p=0.0008$ respectively): 15/28 CE dogs (54\%) were perceived to have fair to poor QOL. There was a strong negative correlation between owner perceived pain and QOL ( $r=-0.81, p<0.05)$. No significant correlation was found between praying position frequency and $\mathrm{mCBPI}$ $(r=0.31, p=0.09)$ or between $\mathrm{mCBPI}$ and mCSUCCPS $(r=0.30, p=0.11)$.

\section{STATEMENT (CONCLUSIONS)}

Visceral pain is an important feature of canine CE, which might not readily detected on physical examination. 


\section{Oral presentations}

It significantly impacts on owner's perception of $\mathrm{QOL}$. This could lead to reduced treatment compliance or euthanasia of patients. Assessment and potential treatment of visceral pain in canine CE demands further study.

\section{Combined endoscopic and fluoroscopic assisted balloon dilation as a treatment method for benign oesophageal strictures in dogs}

\section{Mariette Pilot, Jen O'Keeffe, Gerard Mclauchlan}

Fitzpatrick Referral Oncology \& Soft Tissue, Guildford, United Kingdom

\section{OBJECTIVES}

To assess the effectiveness of combined endoscopic and fluoroscopic assisted balloon dilation in dogs with benign oesophageal strictures.

\section{METHODS}

A retrospective study was performed in dogs that underwent combined endoscopic and fluoroscopic assisted balloon dilation for benign oesophageal strictures. Cases were assigned a Modified Dysphagia Score (MDS) at presentation based on previously published literature (MDS $=0$ if no dysphagia present with normal diet; MDS = 1 if able to swallow some solid food; MDS = 2 if able to swallow semi-solid food; MDS = 3 if able to swallow liquids only; MDS $=4$ if unable to swallow liquids).

Information recorded included the location and number of strictures, duration of clinical signs, fluoroscopic confirmation of full stricture dilation, number of balloon dilations performed and final MDS score. Cases were excluded if assigning a MDS (either pre or post procedure) was not possible due to lack of information in the medical record.

\section{RESULTS}

Five dogs were included in the study. One patient had undergone 4 endoscopic balloon dilations prior to referral. Combined endoscopic and fluoroscopic dilatation was well tolerated in all cases with no complications reported. Patients underwent an average of 2 dilation procedures and all patients were discharged within 24 hours of treatment. The MDS improved in 4/5 cases and remained static in the remaining case. The mean pre treatment MDS was 2 and mean post-treatment MDS was 0.4 .

\section{STATEMENT (CONCLUSIONS)}

Combined endoscopic and fluoroscopic assisted balloon dilation appears an effective method of treating benign oesophageal strictures in dogs.
Indications for administration of gastroprotectant medications in hospitalised dogs and cats

\section{Rachel McCormack ${ }^{1}$, James Swann², Barbara Glanemann ${ }^{2}$}

1 Queen Mother Hospital for Small Animals, Royal Veterinary College, University of London, North Mymms, United Kingdom

2 Dep. of CSS, Royal Veterinary College, University of London, North Mymms, United Kingdom

\section{OBJECTIVES}

To establish how frequently gastroprotectant medications are administered in a specialist veterinary hospital and the stated indications for their use.

\section{METHODS}

A prospective, nurse-led survey was performed over a period of 9 weeks, including all dogs and cats referred to a specialist internal medicine clinic that were hospitalised for more than 24 hours. The use and dosages of gastroprotectant drugs were recorded; attending clinicians completed a questionnaire to establish indications for their use.

\section{RESULTS}

Of 189 dogs and 58 cats hospitalised for more than 24 hours, $74(39 \%)$ dogs and $24(41 \%)$ cats received gastroprotectant drugs. Omeprazole was the drug administered most frequently in 50 dogs and 8 cats, all at a dose 